http://jmscr.igmpublication.org/home/ ISSN (e)-2347-176x ISSN (p) 2455-0450 crossref DOI: https://dx.doi.org/10.18535/jmscr/v9i7.05

\author{
Journal Of Medical Science And Clinical Research \\ IGM Publication \\ An official Publication of IGM Publication
}

\title{
Role of Electroencephalogram and Neuroimaging in Pediatric Seizure Disorder
}

Authors

\section{Dr Kriti Nautiyal ${ }^{1}$, Dr Nita R Sutay ${ }^{2}$, Dr Muthu Vijayanathan ${ }^{3}$, Dr Jyothi Janardhanan ${ }^{4}$, Dr Sangeeta Das ${ }^{5}$}

${ }^{1}$ M.D Pediatrics, Assistant professor, Department of paediatrics, Government Grant Medical College, Sir, J.J hospital, Mumbai

${ }^{2}$ Fellowship in pediatric neurology(MUHS), M.D Pediatrics, Head of Department, Department of paediatrics, Government Grant Medical College, Sir, J.J hospital, Mumbai

${ }^{3}$ M.D Pediatrics, Assistant professor, Department of paediatrics, Government Grant Medical College, Sir, J.J hospital, Mumbai

${ }^{4}$ M.D Pediatrics, Assistant professor, Department of paediatrics, Government Grant Medical College, Sir, J.J hospital, Mumbai

${ }^{5}$ M.D Pediatrics, Assistant professor, Department of paediatrics, Government Grant Medical College, Sir, J.J hospital, Mumbai

\begin{abstract}
Objective: To investigate the frequency of abnormal Neuroimaging and abnormal electroencephalogram findings in pediatric seizure disorder

Methods: A total of 153 patients (ages between 1 month to 12 years) were included from inpatient, outpatient and emergency wards and evaluated as per standardized protocol over a period of 6 months . Seizure pattern was observed and routine investigations sent. CT scan was done in $93.4 \%$, MRI was done in $91.5 \%$, and EEG was done in $83.6 \%$ patients. Patients were categorized into provoked and unprovoked seizure, later investigations results were analysed in both groups

Results: Amongst the studied population generalized tonic clonic seizure was the most commonly observed seizure. MRI was found to be abnormal in $62.8 \%$ patients. EEG showed significant abnormality in $51.5 \%$. Abnormal CT scan only in 46.1\%. EEG and MRI were both found abnormal in $40.6 \%$ patients. There was a statistical significance between EEG and MRI at 99 CI ( $p<0.0001)$.

Conclusion: MRI and EEG form an essential part of evaluation for pediatric seizure disorder and often provide a deeper insight into the underlying abnormality. MRI is more sensitive for yield of white matter abnormalities than CT scan.
\end{abstract}

\section{Introduction}

Epilepsy is a disorder of the brain with enduring predisposition to generate seizures.

A meta-analysis suggests that prevalence of pediatric epilepsy in India is $5.59 / 1000$ with no gender or geographical variations ${ }^{1}$ Occurrence of at least two unprovoked seizures or reflex seizures more than 24 hours apart or one unprovoked epileptic or reflex seizure and a probability (repeat seizure or enough EEG evidence) of further seizure similar to general recurrence risk (at least $60 \%$ ) after two unprovoked seizures, occurring over the next 10 years or diagnosis of an epilepsy syndrome ${ }^{1}$ The American academy of neurology recommends regarding evaluation of first non-febrile seizure urgent Neuroimaging for children with postictal neurological deficit and a routine EEG for all children $^{2}$ 
The current study aims to evaluate the various etiologies of seizure disorder in tric population with the help of Neuroimaging and electroencephalogram findings.

\section{Methods}

This study on the role of EEG and Neuroimaging in pediatric seizure disorder in a tertiary care center on 153 patients. The study was initiated after approval by the Institutional ethical committee. Well informed written consent was obtained from parents or caregivers.

A detailed history of seizure semiology was taken and relevant past history (with or without history of previous seizure), birth history, developmental history, immunization history, anti-epileptic medication history, family history was taken into account and recorded according to the format of proforma designed for this study Patients were later classified into types of seizure based on onset as per the International league against epilepsy classification (2017).

Anthropometric analysis was done with the help of standard WHO age based growth charts. Developmental assessment was done based on developmental history and the developmental quotient was sought in all the 4 domains of milestones: gross motor, fine motor, language milestones, social and adaptive milestones. Neurological assessment included examination of the gait, cranial nerves, motor system, sensory system, cerebellar signs and signs of meningeal irritation. Neurologic examination was classified as abnormal or normal.

Laboratory investigations like serum sodium, potassium, total calcium, (facility of ionic calcium not available at our center), magnesium and random blood sugar were analyzed to look for common metabolic causes of provoked seizure.

Cerebrospinal fluid analysis was done for patients with complaints of fever, single/multiple seizure, altered sensorium, lethargy, vomiting, headache, increasing head size, only after documentation of a normal fundus or no evidence of raised intracranial pressure on CT scan.
CT scan was done only in emergency conditions in children with postictal neurological deficit or in patients suspected to have inflammatory granulomas, or in patients in which risk of prolonged sedation for MRI could not be taken.

SLEEP EEG and MRI was advised in all cases except first episode of simple febrile seizure. ${ }^{1}$

EEG was done using the 10-20 international system of electrodes, using (model) from EB neuro system. EEG was done for the duration of 30 minutes. Hyperventilation and photic stimulation was given. EEG was done within a duration of 4 weeks from seizure episode. EEG reporting was done by the neurologist in our tertiary care center. A normal interictal sleep EEG did not rule out epilepsy.

MRI:- After confirmation, patients were questioned about the history of intracranial surgical clips, cardiac pacemakers, cochlear implants and metallic objects in the body before MR examination.

MRI was performed in all patients using the standardized pediatric seizure protocol and images were analyzed by experienced radiologists at our center

\section{Results}

Table 1: Frequency distribution of study population based on types of focal seizures

\begin{tabular}{|l|c|c|}
\hline $\begin{array}{l}\text { Type of focal } \\
\text { seizures }\end{array}$ & Frequency (n) & $\begin{array}{c}\text { Percentage } \\
(\%)\end{array}$ \\
\hline Tonic clonic & 20 & 13.1 \\
\hline $\begin{array}{l}\text { Focal to } \\
\text { bilateral tonic } \\
\text { clonic }\end{array}$ & 9 & 5.9 \\
\hline Total & 29 & 19 \\
\hline
\end{tabular}

Table 2: Frequency distribution of study population based on types of generalized seizures

\begin{tabular}{|l|c|c|}
\hline $\begin{array}{l}\text { Type } \\
\text { Generalized } \\
\text { seizures }\end{array}$ & $\begin{array}{c}\text { Frequency } \\
\text { (n) }\end{array}$ & $\begin{array}{c}\text { Percentage } \\
(\%)\end{array}$ \\
\hline Absence & 1 & 0.7 \\
\hline Astatic & 3 & 1.9 \\
\hline $\begin{array}{l}\text { Infantile } \\
\text { spasms }\end{array}$ & 4 & 2.6 \\
\hline Myoclonic & 4 & 2.6 \\
\hline Tonic & 43 & 28.8 \\
\hline Tonic Clonic & 68 & 44.4 \\
\hline None & 29 & 18.9 \\
\hline Total & 153 & 100.0 \\
\hline
\end{tabular}


Frequency of provoked and unprovoked seizure were $75(49.1 \%)$ and $78(50.9 \%)$ respectively. EEG an MRI and CT results were studied in these two categories groups.

Global developmental delay was present in $58(37.99 \%)$ patients. Global developmental delay was seen $(24.1 \%)$ in provoked seizure and $(75.8 \%)$ patients in unprovoked seizure.

EEG was available in 128 patients, $51.5 \%$ showed abnormal results. In provoked seizure EEG abnormal in $(25.8 \%)$ patients In unprovoked seizure EEG was abnormal in (72.8\%).

Abnormal EEG findings were as seen follows in our study, an abnormal background was observed in 14 patients $(21.2 \%)$. Amongst epileptiform discharges non-specific epileptiform discharges were seen in (25.7\%) patients and specific abnormal discharges were categorized as spike wave discharges (45.4\%), slow wave discharges in $13.6 \%$ patients, polyspikes in $(12.12 \%)$ and sharp wave discharges (10.6\%).

There was statistical significance observed in EEG findings of both unprovoked and provoked seizure at 95 percent confidence interval $\left(\chi^{2=28.0482}, \mathrm{p}\right.$ value $<.00001)$.

Table 3: EEG abnormalities

\begin{tabular}{|l|c|c|}
\hline $\begin{array}{l}\text { EEG } \\
\text { abnormalities(n=85) }\end{array}$ & Frequency & percentage \\
\hline Abnormal background & 14 & $16.4 \%$ \\
\hline $\begin{array}{l}\text { Epileptiform } \\
\text { abnormalities }\end{array}$ & 71 & $83.5 \%$ \\
\hline -Spike and wave & 30 & $35.2 \%$ \\
\hline -polyspikes & 8 & $9.4 \%$ \\
\hline -Slow wave discharges & 9 & $11.1 \%$ \\
\hline -Sharp wave discharges & 7 & $8.2 \%$ \\
\hline $\begin{array}{l}\text {-Non specified } \\
\text { epileptiform activities }\end{array}$ & 17 & $20 \%$ \\
\hline
\end{tabular}

In our study both abnormal EEG and MRI was seen in (unprovoked: 40 versus provoked:12) total of (40.6\%). Doescher et al. ${ }^{58}$ The EEG and MRI both showed abnormal results in $(21 \%)$ patients.

In our study statistical significance was found between EEG and MRI at 99 percent confidence interval $\left(\chi 2^{=} 27.251^{\mathrm{a}}, \mathrm{p}<0.0001\right)$

\section{MRI Findings}

Table 4: Distribution of abnormalities observed in MRI

\begin{tabular}{|l|c|c|}
\hline Sr.no & MRI finding & No. \\
\hline $\mathbf{1 .}$ & Cystic encephalomalcia & 14 \\
\hline $\mathbf{2 .}$ & Gliosis & 15 \\
\hline $\mathbf{3 .}$ & Leukomalacia & 4 \\
\hline $\mathbf{4 .}$ & Cortical atrophy & 12 \\
\hline $\mathbf{5 .}$ & $\begin{array}{c}\text { Basal ganglia and other subcortical } \\
\text { atrophy }\end{array}$ & \\
\hline $\mathbf{6 .}$ & Mesial sclerosis & 5 \\
\hline $\mathbf{7 .}$ & Tuberculoma/ NCC & 14 \\
\hline $\mathbf{8 .}$ & Beningn cystic lesion & 3 \\
\hline $\mathbf{9 .}$ & Metastatic lesion & 2 \\
\hline $\mathbf{1 0 .}$ & Cerebral dysgenesis & 2 \\
\hline $\mathbf{1 1 .}$ & Chronic hemorrhage & 1 \\
\hline $\mathbf{1 2}$ & Acute hemorrahge & 3 \\
\hline $\mathbf{1 3}$. & Chronic infarct & 2 \\
\hline $\mathbf{1 4}$. & Acute infarct & 6 \\
\hline $\mathbf{1 5}$. & Thrombosis & 3 \\
\hline $\mathbf{1 6}$. & Communicating Hydrocephalus & 10 \\
\hline $\mathbf{1 7 .}$ & Non-communicating Hydrocephalus & 4 \\
\hline $\mathbf{1 8}$. & White matter except periventricular & \\
& leukomalacia & 9 \\
\hline $\mathbf{1 9}$. & Leptomeningitis & 5 \\
\hline $\mathbf{2 0}$. & Pachymeningitis & 1 \\
\hline $\mathbf{2 1 .}$ & Encephalitis & 3 \\
\hline $\mathbf{2 2 .}$ & Ventriculitis & 1 \\
\hline
\end{tabular}

Table no.5: Distribution of abnormalities observed in CT scan

\begin{tabular}{|l|c|c|}
\hline 1. & Cystic encephalomalacia & 10 \\
\hline $\mathbf{2 .}$ & Gliosis & 8 \\
\hline $\mathbf{3 .}$ & Leukomalacia & 3 \\
\hline $\mathbf{4 .}$ & Basal ganglia and other subcortical & \\
& volume loss & 0 \\
\hline $\mathbf{5 .}$ & Cortical volume loss & 13 \\
\hline $\mathbf{6 .}$ & Tuberculoma/ ncc & 13 \\
\hline $\mathbf{7 .}$ & Benign Cystic lesion & 4 \\
\hline $\mathbf{8 .}$ & Mesial sclerosis & 0 \\
\hline $\mathbf{9 .}$ & Metastatic lesion & 2 \\
\hline $\mathbf{1 0 .}$ & Cerebral dysgenesis & 5 \\
\hline $\mathbf{1 1 .}$ & Chronic hemorrhage & 0 \\
\hline $\mathbf{1 2}$ & Acute hemorrhage & 5 \\
\hline $\mathbf{1 3}$. & Chronic infarct & 0 \\
\hline $\mathbf{1 4}$. & Acute infarct & 4 \\
\hline $\mathbf{1 5}$. & Thrombosis & 1 \\
\hline $\mathbf{1 6}$. & Communicating hydrocephalous & 12 \\
\hline $\mathbf{1 7 .}$ & Non-communicating hydrocephalous & 1 \\
\hline $\mathbf{1 8}$. & White matter abnormality except & \\
& periventricular leukomalacia & 0 \\
\hline $\mathbf{1 9}$. & Non specific hypodensities & 1 \\
\hline $\mathbf{2 0}$. & Leptomeningitis & 4 \\
\hline $\mathbf{2 1}$. & Pachymeningitis & 0 \\
\hline $\mathbf{2 2}$. & Enchephalitis & 0 \\
\hline $\mathbf{2 3}$. & Ventriculitis & 0 \\
\hline
\end{tabular}


Unprovoked seizures with abnormal MRI were 75.6\%. Provoked seizures with abnormal MRI were $48.4 \%$. Unprovoked seizure with abnormal CT scan were $52.1 \%$

Provoked seizure with abnormal CT scan were 40.2\%.CT scan and MRI, both neuroimaging modalities were equivocal in radiological diagnosis of hypoxic-ischemic changes, hydrocephalus, benign cystic lesions, inflammatory granulomas, hemorrhage and infarct.

Radiological abnormalities like white matter changes except periventricular leukomalacia, basal ganglia abnormalities, mesial temporal sclerosis were missed on CT scan but gathered only on MRI.

There was statistical significance observed in MRI findings of both unprovoked and provoked seizure at 95 percent confidence interval. $\left(\chi^{2=11.0475}, \mathrm{p}\right.$ value $<.000888$ )

\section{Discussion}

Results showed that a substantial proportion of pediatric seizures present between 1 month-12 months $(62.7 \%)$.Generalized tonic clonic was the most commonly reported seizure $(44.4 \%)$. Patients who had unprovoked seizures were more likely to have EEG and MRI abnormalities. There was no facility of video-EEG or prolonged EEG hence positive yield of EEG abnormality could have been affected.

In our study both abnormal EEG and MRI were seen in a total of $(40.6 \%)$ patients comparable to a similar study of Doescher et al. ${ }^{3}$ The EEG and MRI both showed abnormal results in $(21 \%)$ patients.

The EEG abnormalities most commonly found in our study was comparable to Shinnar et $\mathrm{al}^{4}$. It was also found that abnormal EEGs were more common in partial seizures $(56 \%)$ than in generalized seizures $(35 \%)(\mathrm{p}<0.001)$.

In our study it was observed that cases with unprovoked seizures had abnormal EEG in $(68.3 \%)$. Abnormal EEGs were found in more number in generalized seizure $(75.7 \%)$ than focal seizure (6.1\%)

There were 6 patients in which EEG-specific diagnosis could be made, which were namely
Benign rolandic epilepsy. One patient had classical hypsarrhythmia diagnosed to be West syndrome. One patient was diagnosed to have infantile epileptic encephalopathy. Progressive myoclonic epilepsy, Diffuse encephalopathy in a patient of Aicardi syndrome, Electrical status epilepticus in a patient of were diagnosis in other patients respectively, 2 patients one who had classical Rademaker complexes and another patient had EEG picture like epileptic encephalopathy were later diagnosed as sub-acute pan sclerosing encephalitis.

MRI hold superior yield to CT scan. The most common abnormality being HIE specific changes in $33(23.57 \%)$, volume loss in $18(12.8 \%)$, benign cystic lesion 4(2.8\%), metastatic lesion 1(0.7\%), mesial temporal sclerosis $5(3.5 \%)$, hydrocephalus in $14(10 \%)$, cerebral dysgenesis $5(3.5 \%)$, inflammatory/calcified granulomatous lesions in 14(10\%), white matter lesions except periventricular leukomalacia $9(6.42 \%)$, vascular lesions i.e. haemorrhage $4(2.8 \%)$, infarct $8(5.7 \%)$, thrombosis $3(2.1 \%)$ and inflammation $10(7.1 \%)$. Cerebral dysgenesis recognised on MRI beautifully were namely- Dysembryoplastic neuroepithelial tumor seen as only nonspecific hypodensities on CT scan, Aicardi syndrome, Dyke-Davidoff Masson syndrome, 1 patient with pachygyria and polymicrogyria sequence, 1 patient with only polymicrogyria and 1 patients with Arnold chiari-2 malformation.

Our study MRI results of ventricular enlargement, cortical dysgenesis and hippocampal sclerosis and white matter abnormality was comparable to study. ${ }^{3}$. However proportion of hypoxic ischemic changes and inflammatory/ calcified granulomas was found higher in our study, probably attributable to the socio-economic status of the nation. In the study of Andrew J. Kalnin et $\mathrm{al}^{5}$

leukomalacia/gliosis (23\%) in our study leukomalacia was seen in only $(8.5 \%)$ but encephalomalacia (6\%), in our study seen in $(10 \%)$, but heterotopias and cortical dysplasia were $(12 \%)$ higher percentage than found in our study population. 
Our study attempted to acquire data on both neuroimaging and electroencephalograph in both provoked and unprovoked seizure and since there are population variations our data cannot be easily compared with the other studies.

Not all cases follow up for MRI and EEG. Our data was also dependent upon the recollection of parents or witnesses. Moreover a long term follow up can throw more light on patients whose exact diagnosis remains undetermined.

\section{References}

1. Sridharan R, Murthy BN. Prevalence and pattern of epilepsy in India. Epilepsia 1999;40 631- 636.

2. Hirtz D, Ashwal S, Berg A, Bettis D, Camfield C, Camfield P, Crumrine P, Elterman R, Schneider S, Shinnar S.et al. Practice parameter: evaluating a first nonfebrile seizure in children: report of the quality standards subcommittee of the American Academy of Neurology, the Child Neurology Society, and the American Epilepsy Society. Neurology 2000; Sep 12; 55(5):616-23.

3. Jason. S. dosecher A study done on Magnetic Resonance Imaging and Electroencephalographic Findings in a Cohort of Normal Children With Newly Diagnosed Seizures. J Child Neurol. 2006 Jun; 21(6): 491-495.

4. Shinnar S, Kang H, Berg AT, Goldensohn ES, Hauser WA, Moshe SL. EEG abnormalities in children with a first unprovoked seizure. Epilepsia. 1994;35:471-6. [PubMed

5. Andrew J. Kalnin, MD, Philip S. Fastenau, $\mathrm{PhD}$, Joan K. Austin, DNS. MR Imaging Findings in Children with First Recognized Seizure. Pediatric Neurology, 01 Dec 2008, 39(6):404-41.

6. T. Dura'-Trave'a, M. E. Yoldi-Petria, J. Esparza-Estau' nb, F. Gallinas-Victorianoa, S. Aguilera- Albesaa and A. SagastibelzaZabaletaa Magnetic resonance imaging abnormalities in children with epilepsy European Journal of Neurology 2012, 19:

7. D T. Hsieh, T Chang, T N. Tsuchida, L G. Vezina,Aanderver, J Siedel on New-onset afebrile seizures in infants, Role of neuroimaging. Neurology. 2010 Jan 12; 74(2): 150-156.

8. Shinnar S, Kang H, Berg AT, Goldensohn ES, Hauser WA, Moshe SL. EEG abnormalities in children with a first unprovoked seizure. Epilepsia. 1994;35:471-6. [PubMed]

9. Shinnar S, Berg AT, Moshe SL, et al. The risk of seizure recurrence after a first unprovoked afebrile seizure in childhood: an extended follow-up. Pediatrics. 1996;98:216-25. [PubMed]

10. Berg AT, Shinnar S. The risk of seizure recurrence following a first unprovoked seizure: a quantitative review. Neurology. 1991;41:965-72. [PubMed]. 